\title{
Multilevel perspectives in clinical learning environments' assessment: An insight on levels involved in planning nursing education
}

\author{
Marco Tomietto $^{1}$, Dania Comparcini ${ }^{2}$, Mikko Saarikoski ${ }^{3}$, Valentina Simonetti ${ }^{2}$, Giancarlo \\ Cicolini $^{2-4}$
}

1. University of Udine, Udine, Italy. 2. University “G. D'Annunzio", Chieti, Italy. 3. University of Turku, Turku, Finland. 4. Local Health Provider of Lanciano-Vasto-Chieti, Italy.

Correspondence: Marco Tomietto. Address: School of Nursing, University of Udine, Viale Ungheria, Udine, Italy. Email: marco.tomietto@uniud.it

Received: July 25, 2014

DOI : $10.5430 /$ jnep.v4n12p42
Accepted: September 16, 2014 Online Published: September 27, 2014

URL: http://dx.doi.org/10.5430/jnep.v4n12p42

\section{Abstract}

Background: Clinical learning in nursing education is workplace-based and it involves both learning and organizational factors. Students could experience these factors both at individual and at group level. This study aimed to assess clinical learning environment as a multilevel phenomenon, by grouping students' perceptions at different organizational levels.

Method: A cross-sectional multilevel design has been conducted. 3 Italian Universities, 6 Hospitals and 73 wards have been involved in the study during 2013. Wards with at least 3 attending nursing students have been included. The sample involved 597 nursing students (average age 23.1 years, $\mathrm{SD}=4.67$ years; $72.6 \%$ females; $27.2 \%$ attending first year; $31.7 \%$ second year; $41.1 \%$ third year). Clinical Learning Environment and Supervision plus Nurse teacher (CLES+T) scale has been administered. Intraclass Correlation Coefficients (ICC) have been estimated at ward, at hospital and at University level.

Results: All ICCs scores were above 0.10 and they indicated clinical learning environment as a multilevel phenomenon. The most pertinent level to multilevel research was the ward level. The nurse teacher scale was pertinent to Hospital and University level.

Conclusions: Clinical learning environment is a multilevel phenomenon. These findings could enhance research development in this field of studies. Practical implications suggest multilevel approach in order to detect the most effective organizational level to improve educational intervention.

\section{Key words}

Clinical learning environment, Clinical Learning Environment and Supervision plus Nurse teacher scale, Nursing, Education, Multilevel research 


\section{I ntroduction}

Clinical learning and competences development are essential parts of the nursing curriculum ${ }^{[1]}$. In Europe the percentage of hours spent in clinical training varies from $33 \%$ to $55 \%{ }^{[2]}$. These data highlight the importance of planning clinical experiences to improve students' competences development and to integrate practice and theory ${ }^{[3]}$.

A clinical learning environment is defined as "an interactive network of forces within the clinical setting that influence the students' learning outcomes" ${ }^{[4]}$. So, identifying the forces involved in clinical settings and developing quality indicators of such forces is crucial to improve clinical learning and nursing students' professional growth ${ }^{[5]}$. Moreover, it is important to understand if these forces are an individual perception of each student or if they are a shared perception of a group of students, in order to appropriately set tutorial interventions ${ }^{[6]}$.

Clinical learning environments are multi-dimensional entities, in which many factors are connected and interwoven with each other's. Clinical learning environments are more than pedagogical environments. Factors mentioned in studies are e.g. quality of learning environment, quality of care, nursing staffs' occupational well-being and organizational or managerial variables ${ }^{[7-9]}$. These statements suggest to consider a clinical learning environment wider than only students' training settings.

In this vein, a learning environment was defined as a psyco-social context that, while providing a learning setting, it produces learning itself, and it could be considered as a learning agent ${ }^{[10]}$. Clinical learning is also linked with relational and organizational phenomena within the wards, in addition to pedagogical issues. Undergraduate nurses' clinical experiences are considered as workplace-based learning and they are deeply linked with organizational variables ${ }^{[1]}$.

The forces involved within a clinical setting have been widely explored in research ${ }^{[12-14]}$. In detail, dimensions involved are linked to learning climate within the ward; to organizational variables (such as the role of chief nurse within the ward, the nursing care delivery by the team and the clarity of workflows); to relational variables such as the student-mentor relationship and the nurse teacher role ${ }^{[14]}$. These dimensions are close to the assessment of an organizational climate and, in this vein, they could be approached in a multilevel perspective.

\subsection{Multilevel assessment in clinical learning environments}

A climate could be both an individual perception of an environmental phenomenon and a shared perception of the same phenomenon by the people exposed to the environment ${ }^{[6]}$. This is particularly true in the case of organizational climates, in which it is important to assess a group level perception of the climate to better infer results ${ }^{[15]}$. Under the methodological point of view, the similarity between a clinical learning environment and an organizational environment, suggests to explore the group level perception of nurses students' learning experiences.

Several authors demonstrated that multilevel research is useful to assess nurse practice environment ${ }^{[16]}$, the correlations between work environment and patient safety outcomes ${ }^{[17,18]}$, nurses' burnout ${ }^{[19]}$ and safety climate in the hospital settings ${ }^{[20]}$. In this vein, multilevel modelling is useful to detect the most coherent level to intervene within a workplace (individual, group or organizational level).

Multilevel perspective is theoretically coherent with clinical learning environment research due to the assessment of climate and relational factors. These areas are linked to a shared perception by the students, which attend their clinical placement in the same ward. In fact, students within a same ward are exposed to the same organizational and learning climate. Moreover, they are exposed to the same leadership style of the ward manager and to the same nursing care delivery models. In the same vein, nurse teacher, who is dedicated to the ward in guiding clinical experiences, also tutors a group of students at University level. In this perspective, a clinical learning environment could be coherent with the ward climate, hospital culture and tutorial culture. So it could be useful to assess the hospital level of a clinical learning 
environment perception. Moreover, the nurse teacher role could be differently perceived at University level as well, because each Bachelor could define different tutorial models in guiding students' learning.

According to previous statements this study aimed to demonstrate that a clinical learning environment could be approached as a multilevel phenomenon.

\subsection{Aims and research questions}

This study aimed to assess if a clinical learning environment is a multilevel phenomenon and to assess students' agreement in the evaluation of a clinical learning environment at ward, hospital and University level, by using Clinical Learning Environment and Supervision plus Nurse teacher (CLES+T) scale.

The following research questions have been stated and tested in this study:

- RQ1: Do Intraclass Correlation Coefficients (ICCs) of CLES+T and its factors confirm that a clinical learning environment is a multilevel phenomenon at ward level?

- RQ2: Do Intraclass Correlation Coefficients (ICCs) of CLES+T and its factors confirm that a clinical learning environment is a multilevel phenomenon at hospital level?

- RQ3: Do Intraclass Correlation Coefficients (ICCs) of the Nurse Teacher factor and its sub-factors confirm that nurse teacher role is a multilevel phenomenon at University level?

\section{Methods}

\subsection{Study design}

A cross-sectional study has been conducted. Study was designed in order to aggregate data at ward, at hospital and at University level and to enable multilevel modelling (see Figure 1).

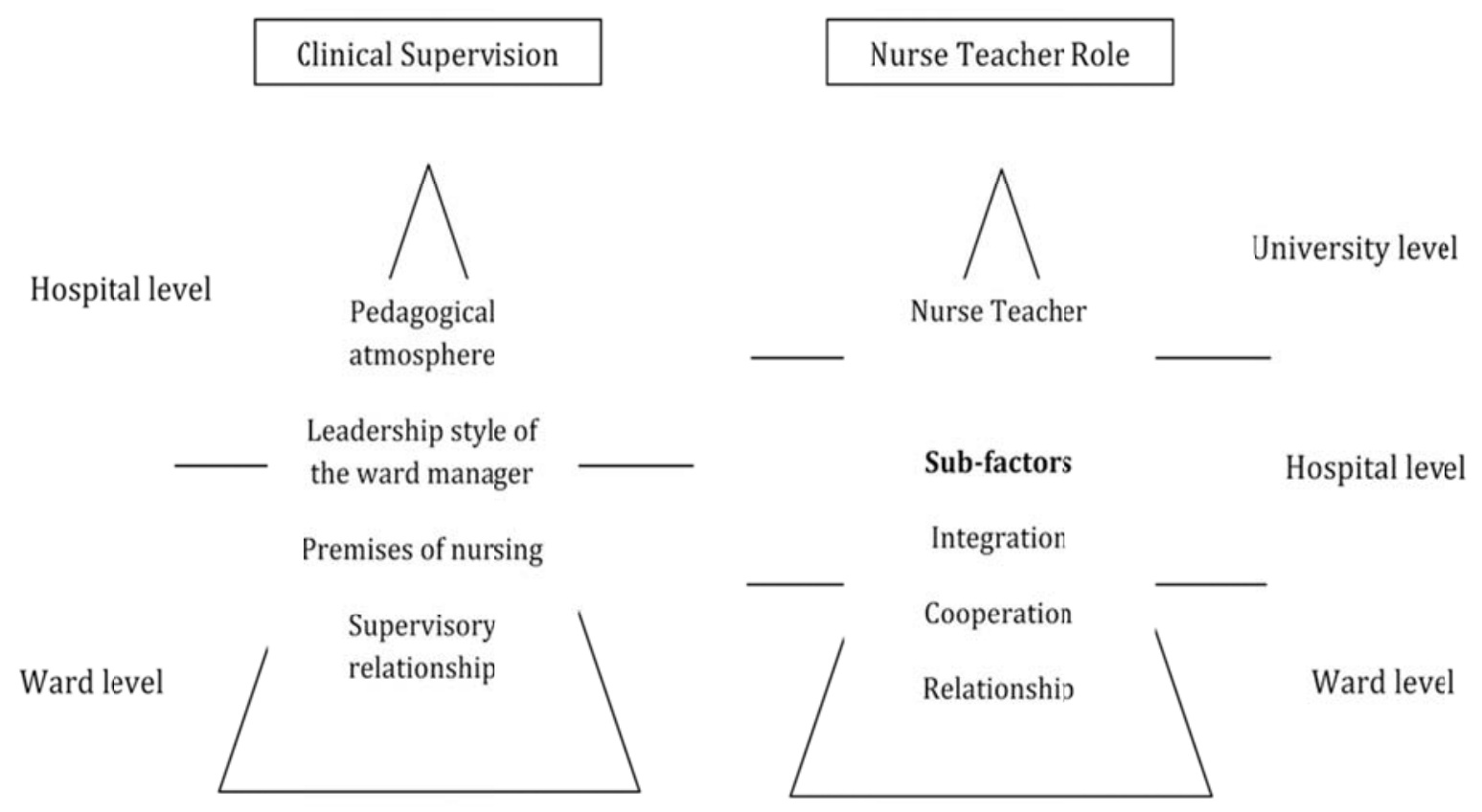

Figure 1. Study model 


\subsection{Participants}

Students attending Nursing Bachelor Degree in 3 Universities in Italy were enrolled in the study with a convenience sampling criterion. All students attending a clinical placement in a hospital ward were eligible to fill CLES+T. In order to perform a group-level analysis, only wards with at least 3 students, which filled CLES+T and which were attending a clinical placement within the same ward, were included in the study ${ }^{[21]}$. Overall, 6 hospitals and 73 wards were involved.

A sample of 597 students participated. The 50.9\% (304/597) of the sample was enrolled in University 1, 165/597 (27.6\%) students in University 2 and 128/597 (21.5\%) students in University 3. Students in University 1 attended their clinical placement in 3 different hospitals (respectively 208, 50 and 46 students), while students in University 2 in 1 hospital (165 students) and students in University 3 in 2 hospitals (respectively 82 and 46 students).

Students attended for the $31.0 \%$ (185/597) a medical ward, for the $28.6 \%(171 / 597)$ a surgical ward, for the $24.3 \%$ $(145 / 597)$ a critical area ward, while the remaining $16.1 \%$ students $(96 / 597)$ attended outpatient services and paediatric wards. Overall, 73 wards were attended by a minimum of 3 to a maximum of 24 students for each ward.

The mean age of the sample was 23.1 years (median 22, SD 4.67, $\min 19$, max 48), 72.6\% (429/597) students were female, while $27.2 \%$ (161/593) were attending the first year, 31.7\% (188/593) the second year and $41.1 \%(244 / 593)$ the third year (4 data missing).

Mean clinical placement duration was 6.0 weeks (median 5, SD 1.75, min 2, max 12).

\subsection{Instruments}

Background variables were included in the questionnaire in order to describe sample and the students' clinical placements and to match CLES+T scores with the specific ward attended by students.

Clinical Learning Environment and Supervision plus nurse Teacher (CLES+T) scale ${ }^{[14,22]}$ has been used to assess students' perception of the clinical learning environment within the ward. Dimensions assessed by CLES+T are: pedagogical atmosphere (9 items concerning students' perception of ward learning climate); leadership style of the ward manager (4 items concerning ward manager's integration in the team); premises of nursing on the ward (4 items which assess how much nursing care is personalized and if nursing documentation is clear); supervisory relationship ( 8 items which explore if mentor-student relationship is characterized by a mutual interaction and it is oriented to student's learning needs) and the nurse teacher scale ( 9 items which assess integration, cooperation and relationship of the nurse teacher with the students and the ward team) ${ }^{[14]}$.

Previous studies have tested the psychometric proprieties of CLES + T scale both internationally ${ }^{[14,23]}$ and in Italian clinical settings ${ }^{[24]}$, demonstrating the validity and reliability of the scale. However, two different models emerged in defining the framework of a clinical learning environment. Both models highlighted psychometric proprieties and reliability indexes, which confirmed CLES $+\mathrm{T}$ scale as the gold standard to assess a clinical learning environment internationally in comparison to other instruments tested ${ }^{[12,13,25]}$. In particular the Italian version, in a validation study involving 875 students, highlighted a Cronbach's alpha coefficient of 0.95 , ranging from 0.80 and 0.96 among factors, stating the instrument's reliability ${ }^{[24]}$. Italian results were similar to the original validation of CLES+T and the variance explained in Exploratory Factor Analysis (EFA) was the same of the study held in Finland. However, the factorial structure highlighted a 7-factor model in spite of a 5-factor model: the nurse teacher scale in the Italian validation was divided in 3 sub-factors by the EFA and it was confirmed as the best valid solution in Confirmatory Factor Analysis (CFA). In detail the "nurse teacher" scale was divided in an "integration" sub-factor (3 items), "cooperation" sub-factor (3 items) and "relationship" sub-factor (3 items) ${ }^{[24]}$. In the Italian validation the most important factor was "pedagogical atmosphere" and the total variance explained by CLES+T scale was $67 \%$. 
In the original study, which recruited 549 students in Finland, CLES+T scale had alpha coefficients ranging from 0.77 to 0.96 and it explained $67 \%$ of variance, with "supervisory relationship" as the main factor $(40 \%){ }^{[14]}$.

In this study both the 7-factors model and the 5-factors model has been tested according to the Italian validation ${ }^{[24]}$ and the International validation results ${ }^{[14]}$. CLES $+\mathrm{T}$ Italian version used in this study had already been translated in previous studies ${ }^{[24,26,27]}$ following back-forward translation criteria in order to ensure content validity.

\subsection{Data collection procedures}

The coordinating centre in Chieti granted the ethical approval. Three trained researchers (one in each university) personally delivered the CLES+T scale from February to July 2013. CLES+T scale was individually administered in the last week of each student's clinical placement, in order to avoid recall bias. Each student received, at the beginning of the clinical placement, an information letter about the CLES+T scale and a reminder in the middle of the training period ${ }^{[28]}$. The researcher asked the students to fill and to return the questionnaires within 20 minutes. To guarantee confidentiality and anonymity, participants re-submitted the questionnaire in an envelope inside a dedicated box within the ward. Restitution of the completed questionnaire was considered as participants' agreement to the study.

\subsection{Data analysis procedures}

Preliminary data analyses were performed in order to assess scale reliability ${ }^{[29]}$. Scale reliability was measured with Cronbach's alpha, while validity was assessed with Confirmatory Factor Analysis ${ }^{[30]}$.

The requirements for multilevel analyses were assessed calculating the Intraclass Correlation Coefficients (ICCs) and their Confidence Intervals at 95\% (CI 95\%). ICC represents the total variability of a variable that is pertinent with grouping. ICC is assumed to be an index of agreement within a group about a given variable, and it represents the convergence of individuals' assessment. If there is a wide variability among individuals within the same group, ICC is small. If individual perceptions within a group are convergent (low variability), ICC is high ${ }^{[31]}$. The acceptable ICC's cut-off value to perform multilevel analyses is 0.10 : higher values confirm that multilevel analyses are pertinent ${ }^{[31]}$.

ICC has been calculated for each factor and for the whole scale at ward and hospital level, the nurse teacher factor and sub-factors have been considered also at University level.

Multilevel approach requires a hieratical grouping ${ }^{[21]}$ in this study two hieratical models have been assumed. The first one regarded the hierarchy in grouping clinical supervision received by students within hospitals. In the first model, each group of students perceived a pedagogical atmosphere within the wards, a leadership style of the ward manager, the premises of nursing care and a relationship with clinical supervisor, which depended by the organizational environment attended (at ward and at hospital level). The second model regarded the hieratical structure in nurse teacher supervision: each group of students perceived a nurse teacher role, which depended by the role acted within the ward, by the hospital but even by the University. In this vein, the nurse teacher factor has been evaluated also at University level, both as a unique factor and both in its 3 sub-factors, according to the psychometric characteristics stated in this study and in literature review (see Figure 1).

Preliminary data analyses were performed with SPSS v20, while ICCs and 95\% CIs were calculated using Stata v12. Confirmatory Factor Analysis was performed with the "sembuilder" package in Stata v12.

\subsection{Ethical considerations}

The Ethical Committee of the University "G. D'Annunzio" in Chieti approved the study before data collection. 


\section{Results}

\subsection{Preliminary analyses}

Number of observations at ward level varied from 3 to 24, at hospital level from 46 to 208 and at University level from 128 to 304 . The variability between groups was significant at each level of assessment $(p=.001)$.

In this study, Cronbach's alpha of CLES+T scale was 0.96. Among factors, Cronbach's alpha was 0.92 in "pedagogical atmosphere", it was 0.87 in "leadership style of the ward manager", it was 0.83 in "premises of nursing in the ward", it was 0.97 in "supervisory relationship" and it was 0.93 in the "nurse teacher integration" sub-factor, 0.91 in the nurse teacher cooperation" sub-factor and 0.94 in the "nurse teacher relationship" sub-factor. Overall, Cronbach's alpha of the "nurse teacher" scale was 0.94 .

CFA in this study confirmed adequate fit indexes for the 7 factors model with RMSEA $=0.067, \mathrm{CFI}=0.927, \mathrm{TLI}=0.919$ and SRMR $=0.046$, while the 5 factors model indicated borderline fit indexes with RMSEA $=0.091, \mathrm{CFI}=0.862$, TLI $=$ 0.850 and $\mathrm{SRMR}=0.051$.

\subsection{Testing multilevel requirements at ward, hospital and university level}

All research aims have been confirmed. In detail, the first research question (RQ1) was about the ward level of students' agreement in clinical learning environment perception. Results indicated that ICC of the "pedagogical atmosphere" factor was $0.48(95 \% \mathrm{CI}=0.38-0.58)$, while in the "leadership style of the ward manager" factor ICC was $0.39(95 \% \mathrm{CI}=$ $0.29-0.49)$, in the "premises of nursing" factor it was $0.33(95 \% \mathrm{CI}=0.24-0.44)$, in the "supervisory relationship" factor it was $0.42(95 \% \mathrm{CI}=0.32-0.53)$. In the overall "nurse teacher" factor, ICC was $0.13(95 \% \mathrm{CI}=0.07-0.22)$ and it varied from 0.09 to 0.11 among sub-factors. Overall ICC of the whole CLES+T scale at ward level was $0.42(95 \% \mathrm{CI}=0.32-0.52)$.

At hospital level (RQ2), "pedagogical atmosphere" factor highlighted an ICC of $0.26(95 \% \mathrm{CI}=0.09-0.52)$, "leadership style of the ward manager" factor of $0.19(95 \% \mathrm{CI}=0.06-0.41)$, "premises of nursing in the ward" of $0.20(95 \% \mathrm{CI}=$ 0.06-0.43), and "supervisory relationship" of $0.24(95 \% \mathrm{CI}=0.11-0.56)$. "Nurse teacher" sub-factors' ICCs varied from 0.10 to 0.16 , while the overall factor had an ICC of $0.17(95 \% \mathrm{CI}=0.05-0.40)$. CLES+T scale at hospital level had an ICC of $0.28(95 \% \mathrm{CI}=0.01-0.54)$.

The third research question explored the "nurse teacher" factor and its sub-factors at University level. Results stated an ICC of $0.14(95 \% \mathrm{CI}=0.02-0.43)$ in the whole factor, and, in detail, ICC was $0.10(95 \% \mathrm{CI}=0.01-0.33)$ in the "integration" sub-factor, while in the "cooperation" sub-factor it was $0.13(95 \% \mathrm{CI}=0.02-0.40)$ and it was $0.10(95 \% \mathrm{CI}=0.01-0.34)$ in the "relationship" sub-factor.

It's possible to notice that the ward level had lower standard errors (SEs) and narrower 95\% CIs than hospital and University level. At ward level, none of the CIs' lower limits were under 0.10 (from 0.24 to 0.32 ), while upper limits ranged from 0.44 to 0.58 .

All ICCs were over the 0.10 cut-off value, however NT factor and sub-factors indicated borderline values and $95 \%$ CIs lower limit ranged from 0.01 to 0.07 and the upper limit from 0.18 to 0.43 . At hospital level the lower limit ranged from 0.01 to 0.11 and the upper limit from 0.41 to 0.56 . In the same vein, SEs at ward level are $\leq 0.05$ and at hospital level $\geq 0.06$. Table 1 represents the detailed results about ICCs, SEs, 95\% CIs. 
Table 1. ICCs at different levels of analysis in CLES+T scale, factors and sub-factors

\begin{tabular}{|c|c|c|c|c|c|c|c|}
\hline Factors - subfactors - scale & Level & min-max obs & ICC & SE & $95 \%$ CI & Wald z & $p$ \\
\hline \multirow{2}{*}{ Pedagogical atmosphere } & Ward & $3-24$ & 0.48 & 0.05 & $0.38-0.58$ & 53.10 & .001 \\
\hline & Hospital & $46-208$ & 0.26 & 0.11 & $0.09-0.52$ & 23.87 & .001 \\
\hline \multirow{2}{*}{$\begin{array}{l}\text { Leadership style of the ward } \\
\text { manager }\end{array}$} & Ward & $3-24$ & 0.39 & 0.05 & $0.29-0.49$ & 45.53 & .001 \\
\hline & Hospital & $46-208$ & 0.19 & 0.09 & $0.06-0.41$ & 22.00 & .001 \\
\hline \multirow{2}{*}{ Premises of nursing in the ward } & Ward & $3-24$ & 0.33 & 0.05 & $0.24-0.44$ & 56.99 & .001 \\
\hline & Hospital & $46-208$ & 0.20 & 0.09 & $0.06-0.43$ & 25.31 & .001 \\
\hline \multirow{2}{*}{ Supervisory relationship } & Ward & $3-24$ & 0.42 & 0.05 & $0.32-0.53$ & 37.83 & .001 \\
\hline & Hospital & $46-208$ & 0.29 & 0.12 & $0.11-0.56$ & 15.83 & .001 \\
\hline \multirow{3}{*}{ Nurse Teacher - integration } & Ward & $3-24$ & 0.10 & 0.03 & $0.05-0.19$ & 53.83 & .001 \\
\hline & Hospital & $46-208$ & 0.10 & 0.06 & $0.02-0.27$ & 22.25 & .001 \\
\hline & University & $128-304$ & 0.10 & 0.08 & $0.01-0.33$ & 16.44 & .001 \\
\hline \multirow{3}{*}{ Nurse Teacher - cooperation } & Ward & $3-24$ & 0.11 & 0.03 & $0.06-0.20$ & 38.71 & .001 \\
\hline & Hospital & $46-208$ & 0.16 & 0.09 & $0.05-0.38$ & 13.11 & .001 \\
\hline & University & $128-304$ & 0.13 & 0.09 & $0.02-0.40$ & 10.87 & .001 \\
\hline \multirow{3}{*}{ Nurse Teacher - relationship } & Ward & $3-24$ & 0.09 & 0.03 & $0.04-0.18$ & 42.42 & .001 \\
\hline & Hospital & $46-208$ & 0.14 & 0.08 & $0.04-0.35$ & 14.52 & .001 \\
\hline & University & $128-304$ & 0.10 & 0.08 & $0.01-0.34$ & 12.75 & .001 \\
\hline \multirow{3}{*}{ Nurse Teacher factor } & Ward & $3-24$ & 0.13 & 0.04 & $0.07-0.22$ & 48.35 & .001 \\
\hline & Hospital & $46-208$ & 0.17 & 0.09 & $0.05-0.40$ & 16.13 & .001 \\
\hline & University & $128-304$ & 0.14 & 0.10 & $0.02-0.43$ & 13.19 & .001 \\
\hline \multirow{2}{*}{ CLES+T scale } & Ward & $3-24$ & 0.42 & 0.05 & $0.32-0.52$ & 54.33 & .001 \\
\hline & Hospital & $46-208$ & 0.28 & 0.12 & $0.01-0.54$ & 22.38 & .001 \\
\hline
\end{tabular}

\section{Discussion}

This study highlighted multilevel characteristics of a clinical learning environment. Findings suggested that research and practice in health care education could usefully take into account both the interacting forces and the shared perceptions of such forces within a clinical setting. This approach could be useful to focus on the most appropriate level of intervention in order to improve clinical learning environments attended by students. In detail, the ward level was the most reliable dimension to multilevel approach. This is coherent with previous research about nursing work environments that indicated the ward group as the most suitable level to predict patient safety outcomes ${ }^{[17]}$ as well as nurses wellbeing ${ }^{[18]}$. A clinical learning environment is acted and perceived within an organizational context and, in this vein, it demonstrated similar characteristics of agreement in ward-level grouping. Moreover ward is the closest organizational dimension in students' experience and it is coherent that students perceived this level in a convergent way within each ward.

However, results indicated that the nurse teacher scale and its sub-factors (5-factors model and 7-factors model) are border line to multilevel premises. May be the tutorial relationship between the nurse teacher and the student was perceived as individually built and it had a wide variability even within the same ward. On the contrary, ICCs in the nurse teacher scale and sub-factors tended to be higher moving from the ward level to the hospital or to the University level. In this case the nurse teacher role could be perceived as coherent with a wider organizational dimension and it could be oriented toward a tutorship culture imprinted at hospital and at University level. This seems pertinent with the results by a European comparison of the nurse teacher role ${ }^{[2]}$, in which emerged a wide cross-Country variability in acting the nurse teacher role. In this vein, the nurse teacher role could depend by a wider organizational dimension. 
These findings suggested that the ward level is privileged in multilevel research to study the pedagogical atmosphere, leadership style of the ward manager, premises of nursing and supervisory relationship within a team unit. The hospital and the University levels are more suitable to study nurse teacher role. Under a research perspective, this study highlighted CLES+T as a multilevel scale.

Moreover, this study underlined that multilevel approach could be useful to plan tutorial strategies and interventions in students' clinical placements. Specifically, when a dimension of a clinical learning environment is perceived at ward level, nurse teacher has to closely work with the ward manager in order to build a tutorial climate within the teamwork and to enhance a students' learning-oriented setting. When a dimension is perceived at hospital or at University level, interventions have to focus in designing tutorship models and in building collaborations to develop an effective liaison between academic and organizational settings in managing clinical placements.

\section{Limitations}

The limitations of this study pertain to the evaluation of University and hospital level: ICCs were calculated involving 3 Universities and 6 hospitals. A wider amount of groups in these levels could improve indexes' accuracy and it could generate narrower $95 \%$ CIs and lower SEs, than the ones found in this study ${ }^{[32]}$. It could be interesting to develop this study at international level in order to evaluate also a Country level of agreement, especially about the nurse teacher role, which has demonstrated a wide variability in European Countries ${ }^{[2]}$. This strategy could contribute to better clarify the multilevel characteristics of a clinical learning environment at the hospital and at the University level, which highlighted borderline ICCs and CIs in this study.

\section{Conclusion}

This study stated the multilevel nature of a clinical learning environment and it highlighted CLES+T scale as coherent with multilevel research. While the individual level was widely explored, findings suggested that health care education in clinical settings has to be considered similarly to the organizational variables within nursing environments. In detail, the ward level emerged as the most informative level to go further in this research field.

Multilevel approach could also give an important contribution to set educational interventions in the most pertinent level according to clinical learning environment's dimensions. Ward, hospital and University level act differently in clinical settings and, to recognise differences among these levels, could effectively contribute in guiding students' clinical learning experiences.

\section{References}

[1] Benner P. From novice to expert: Excellence and power in clinical nursing practice. Menlo Park: Addison-Wesley; 1984.

[2] Warne T, et al. An exploration of the clinical learning experience of nursing students in nine European countries. Nurse Education Today. 2010; 30(8): 809-815. PMid:20409620 http://dx.doi.org/10.1016/j.nedt.2010.03.003

[3] Davies C, et al. Teaching in practice. Nursing Standard. 1999; 13(35): 33-35. PMid:10455672 http://dx.doi.org/10.7748/ns1999.05.13.35.33.c2604

[4] Dunn SV, Burnett P. The development of a clinical learning environment scale. Journal of Advanced Nursing. 1995; 22(6): 1166-1173. http://dx.doi.org/10.1111/j.1365-2648.1995.tb03119.x

[5] Lockwood-Rayermann S. Preceptors, leadership style, and the student practicum experience. Nurse Educator. 2003; 28(6): 247-249. PMid:14624120 http://dx.doi.org/10.1097/00006223-200311000-00003

[6] Schneider B, Ehrhart MG, Macey WH. Organizational climate and culture. Annual Review of Psychology. 2013; 64: 361-388. PMid:22856467 http://dx.doi.org/10.1146/annurev-psych-113011-143809

[7] Smith P. The relationship between quality of nursing care and the ward as a learning environment: developing a methodology. Journal of Advanced Nursing. 1987; 12:413-420. PMid:3655130 http://dx.doi.org/10.1111/j.1365-2648.1987.tb01350.x 
[8] Leveck ML, Jones CB. The nursing practice environment, staff retention, and quality of care. Research in Nursing \& Health. 1996; 19: 331-343. http://dx.doi.org/10.1002/(SICI)1098-240X(199608)19:4<331::AID-NUR7>3.0.CO;2-J

[9] Saarikoski M, Leino-Kilpi H. Association between quality of ward nursing care and students' assessment of the ward as a clinical learning environment. NT research. 1999; 4: 467-474. http://dx.doi.org/10.1177/136140969900400611

[10] Gabel D. Handbook of research on science teaching and learning. McMillan Publishing Company, New York: McMillan Publishing Company; 1993.

[11] Bergjan M, Hertel F. Evaluating students' perception of their clinical placements - Testing the clinical learning environment and supervision and nurse teacher scale (CLES+ T scale) in Germany. Nurse Education Today. 2013; 33(11): $1393-1398$. PMid:23200088 http://dx.doi.org/10.1016/j.nedt.2012.11.002

[12] Chan DSK. Combining qualitative and quantitative methods in assessing hospital learning environments. International Journal of Nursing Studies. 2001; 38(4): 447-459. http://dx.doi.org/10.1016/S0020-7489(00)00082-1

[13] Hosoda Y. Development and testing of a Clinical Learning Environment Diagnostic Inventory for baccalaureate nursing students. Journal of Advanced Nursing. 2006; 56(5): 480-490. PMid:17078824 http://dx.doi.org/10.1111/j.1365-2648.2006.04048.x

[14] Saarikoski M, et al. The nurse teacher in clinical practice: developing the new sub-dimension to the Clinical Learning Environment and Supervision (CLES) Scale. International Journal of Nursing Studies. 2008; 45(8): 1233-1237. PMid:17803996 http://dx.doi.org/10.1016/j.ijnurstu.2007.07.009

[15] Zohar D. Thirty years of safety climate research: Reflections and future directions. Accident Analysis \& Prevention. 2010; 42(5): 1517-1522. PMid:20538108 http://dx.doi.org/10.1016/j.aap.2009.12.019

[16] Li B, et al. Group-level impact of work environment dimensions on burnout experiences among nurses: A multivariate multilevel probit model. International Journal of Nursing Studies. 2012; 50: 281-291. PMid:22831971 http://dx.doi.org/10.1016/j.ijnurstu.2012.07.001

[17] Purdy N, et al. Effects of work environments on nurse and patient outcomes. Journal of Nursing Management. 2010; 18(8): 901-913. PMid:21073564 http://dx.doi.org/10.1111/j.1365-2834.2010.01172.x

[18] Van Bogaert P, et al. Impacts of unit-level nurse practice environment, workload and burnout on nurse-reported outcomes in psychiatric hospitals: A multilevel modelling approach. International Journal of Nursing Studies. 2013; 50: 357-365. PMid:22695484 http://dx.doi.org/10.1016/j.ijnurstu.2012.05.006

[19] Kirwan M, Matthews A, Scott PA. The impact of the work environment of nurses on patient safety outcomes: A multi-level modelling approach. International Journal of Nursing Studies. 2012; 50: 253-263. PMid:23116681 http://dx.doi.org/10.1016/j.ijnurstu.2012.08.020

[20] Nieva V, Sorra J. Safety culture assessment: a tool for improving patient safety in healthcare organizations. Quality and Safety in Health Care. 2003; 12(2): 17-23.

[21] Hox JJ. Multilevel analysis: Techniques and applications. Florence: Psychology Press; 2002.

[22] Saarikoski M, Leino-Kilpi H.The clinical learning environment and supervision by staff nurses: developing the instrument. International Journal of Nursing Studies. 2002; 39(3): 259-267. http://dx.doi.org/10.1016/S0020-7489(01)00031-1

[23] Johansson UB, et al. Clinical learning environment, supervision and nurse teacher evaluation scale: psychometric evaluation of the Swedish version. Journal of Advanced Nursing. 2010; 66(9): 2085-2093. PMid:20626485

[24] Tomietto M, et al. Clinical Learning Environment and Supervision plus nurse Teacher (CLES+T) scale: testing the psychometric characteristics of the Italian version. Giornale Italiano di Medicina del Lavoro ed Ergonomia. 2012; 34(2): B72-B80. PMid:23326942

[25] Perli P, Brugnolli A. Italian nursing students' perception of their clinical learning environment as measured with the CLEI tool. Nurse Education Today. 2009; 29(8): 886-890. PMid:19576663 http://dx.doi.org/10.1016/j.nedt.2009.05.016

[26] Tomietto M, et al. La valutazione della qualità degli ambienti di apprendimento clinico: studio di validazione del Clinical Learning Environment and Supervision (CLES) nel contesto italiano. Giornale Italiano di Medicina del Lavoro ed Ergononomia. 2009; 31(3): B49-55.

[27] Comparcini D, et al. Nursing students' satisfaction and perception of their first clinical placement: observational study, Professioni Infermieristiche. 2014; 67(1): 41-7. PMid:24762772

[28] Dillman DA, Smyth JD, Christian LM. Internet, mail, and mixed-mode surveys: The tailored design method. Hoboken: Wiley; 2009.

[29] Barbaranelli C. Analisi dei dati. Tecniche multivariate per la ricerca psicologica e sociale. Milano: LED; 2007.

[30] DeVellis RF. Scale Development: Theory and Applications. Thousand Oaks: SAGE Pubblications; 2011.

[31] Kahn JH. Multilevel modeling: overview and applications to research in counseling psychology. Journal of Counseling Psychology. 2011; 58(2): 257-271. PMid:21463032 http://dx.doi.org/10.1037/a0022680

[32] Maas CJ, Hox JJ. Sufficient sample sizes for multilevel modeling. European Journal of Research Methods for the Behavioral and Social Sciences. 2005; 1(3): 86-92. http://dx.doi.org/10.1027/1614-2241.1.3.86 